\title{
Multi-Hop Clustering Protocol using Gateway Nodes in Wireless Sensor Network
}

\author{
S. Taruna ${ }^{1}$, Rekha Kumawat ${ }^{2}$, G.N.Purohit ${ }^{3}$ \\ ${ }^{1}$ Banasthali University, Jaipur, Rajasthan \\ staruna 71 @yahoo.com \\ ${ }^{2}$ Banasthali University, Jaipur, Rajasthan \\ er.rekha28@gmail.com \\ ${ }^{3}$ Banasthali University, Jaipur, Rajasthan \\ gn_purohitjaipur@yahoo.com
}

\begin{abstract}
Wireless sensor networks (WSNs) are composed of many homogeneous or heterogeneous sensor nodes with limited resources. A sensor node is comprised of three components: a sensor, a processor and a wireless communication device. These sensor nodes dispersed throughout it to monitor, collect, and transmit data. The sensors are inexpensive, simple, and their power source is irreplaceable. Knowing the sensors power levels cannot be restored, many protocols have been developed to make collecting, receiving and transferring data more energy efficient. In this paper, we propose a multi-hop cluster based routing protocol which is more energy efficient than single hop protocol. Simulation results show that the protocol offers a better performance than single-hop clustering routing protocols in terms of network lifetime and energy consumption by improving FND.
\end{abstract}

\section{KEYWORDS}

Wireless Sensor Network, First Node Death (FND), Multi-Hop Communication, Energy Efficient, Gateway Nodes

\section{INTRODUCTION}

Wireless sensor networks (WSN's) [1] have gained worldwide attention in recent years, particularly with the proliferation in Micro-Electro-Mechanical Systems (MEMS) technology which has facilitated the development of smart sensors. These sensors are small, with limited processing and computing resources, and they are inexpensive compared to traditional sensors. These sensor nodes can sense, measure, and gather information from the environment and, based on some local decision process, they can transmit the sensed data to the user.

A WSN typically has little or no infrastructure. It consists of a number of sensor nodes (few tens to thousands) working together to monitor a region to obtain data about the environment. These sensors have the ability to communicate either among each other or directly to an external base-station (BS). A greater number of sensors allows for sensing over larger geographical regions with greater accuracy. The sensor sends such collected data, usually via radio transmitter, to a command center (sink) either directly or through a data concentration center (a gateway). 
Routing protocol is one of the core technologies in the WSN. Due to its inherent characteristics, routing is full of challenge in WSN [2]. Clustering is a well-know and widely used exploratory data analysis technique, and it is particularly useful for applications that require scalability to hundreds or thousands of nodes [3]. For large-scale networks, node clustering has been proposed for efficient organization of the sensor network topology, and prolonging the network lifetime. Among the sources of energy consumption in a sensor node, wireless data transmission is the most critical. Within a clustering organization, intra-cluster communication can be single hop or multi-hop, as well as inter-cluster communication.

In this paper, we analyze energy efficient multi-hop clustering routing algorithm by a sensor node for WSN. We first describe the new energy based multi-hop with gateway node routing scheme, and then simulation results in MATLAB [4]. Further, the performance analysis of the proposed scheme is compared with benchmark clustering algorithm LEACH [5].

The remainder of this paper is organized as follows: Section 2 describes the related work. Section 3 describes the proposed multi-hop routing scheme. Simulation results are discussed in section 4 and conclusions are drawn in section 5 .

\section{RELATED WORKS}

In sensor networks deployed in harsh or unstructured environments, sensor nodes are typically powered by irreplaceable batteries with a limited amount of energy supply. Ideally we would like the sensor network to perform its functionality as long as possible. Optimal routing maximizes the network functionality by minimizing the total energy consumption and optimizing the network-wide load balance to prolong the lifetime of sensor networks have been an essential task in sensor network implementation.

Routing is a process of determining a path between source and destination upon request of data transmission. A variety of protocols have been proposed to enhance the life of WSN and for routing the correct data to the base station. Employing clustering techniques in routing protocols can hierarchically organize the network topology and prolongs the lifetime of a wireless sensor network, and contributes to overall system scalability. Various protocols [6] like LEACH, HEED, PEGASIS, TEEN, and APTEEN are available to route the data from node to base station in WSN.

A single-hop clustering routing protocol can reduce the communication overhead by selecting a $\mathrm{CH}$ to forward data to base station via one hop. Many single-hops clustering routing protocol have been proposed like LEACH and HEED. But when communication distance increases, single hop communication consumes more energy. Multi-hop communication consumes less energy than single hop protocols for long distances. Many multi-hop routing protocols have been proposed like M-LEACH [7] and MR-LEACH [8].

Low Energy Adaptive Clustering Hierarchy (LEACH) is the first clustering protocol that was proposed for reducing power consumption. It forms clusters by using a distributed algorithm, each node has a certain probability of becoming a cluster head per round, and the task of being a cluster head is rotated between nodes. A non-CH node determines its cluster by choosing the $\mathrm{CH}$ that can be reached with the least communication energy consumption. In the data transmission stage, each cluster head sends an aggregated packet to the base station by single hop. 
LEACH randomly selects a few sensor nodes as $\mathrm{CHs}$ and rotates this role to evenly load among the sensors in the network in each round. In LEACH, the cluster head $(\mathrm{CH})$ nodes compress data arriving from nodes that belong to the respective cluster, and send an aggregated packet to the base station. A predetermined fraction of nodes, p, elect themselves as $\mathrm{CHs}$ in the following manner. A sensor node chooses a random number, $r$, between 0 and 1 . If this random number is less than a threshold value, $\mathrm{T}(\mathrm{n})$, the node becomes a cluster-head for the current round. The threshold value is calculated based on an equation that incorporates the desired percentage to become a cluster-head, the current round, and the set of nodes that have not been selected as a cluster-head in the last $(1 / \mathrm{p})$ rounds, denoted by $\mathrm{G}$. It is given by:

$$
T(n)=p / 1-p(r \bmod (1 / p)) \text { if } n € \mathrm{G}
$$

Each elected $\mathrm{CH}$ broadcasts an advertisement message to the rest of the nodes in the network that they are the new cluster-heads. A sensor node or non- $\mathrm{CH}$ selects the $\mathrm{CHs}$ which is nearest to it.

Inter-Intra Cluster Multi hop-LEACH (M-LEACH) [7] is a cluster based routing algorithm. Basic operation of Multi hop-LEACH is similar to LEACH protocol. There are two major modifications in Multi hop-LEACH protocol with respect to LEACH protocol. Multi hoping is applied to both inter cluster and intra cluster communication. Each cluster is composed of one cluster head $(\mathrm{CH})$ and cluster member nodes. The respective $\mathrm{CH}$ gets the sensed data from its cluster member nodes, aggregates the sensed information and then sends it to the Base Station through an optimal multi-hop tree formed between cluster heads (CHs) with base station as root node. When the sensor nodes are deployed in regions of dense vegetation or uneven terrain, it may be beneficial to use multi-hop communication among the nodes in the cluster to reach the cluster head. Intra cluster communication performs in the same way like inter cluster communication.

\section{The Proposed Multi-hop Algorithm}

Basic operation of proposed multi-hop clustering routing protocol is multi-hop transmission of data from $\mathrm{CH}$ to BS. A multilevel hierarchical, data gathering sensor network architecture is used in this scheme. At the lowest level sensor nodes send data to cluster head, and cluster head sends data to gateway nodes. The gateway nodes, which forms the next level of hierarchy, are programmed to communicate with a sink (Base station) located outside from the network field.

\subsection{Assumptions}

The following assumptions are made for the new scheme:

- The Base Station (i.e. data sink) located far away from the sensing field and it is stationary after deployment.

- The Base station (BS) has the information about the location of each node and the location of gateway nodes.

- Nodes are dispersed in a 2-dimensional space and cannot be recharged after deployment.

- Nodes are uniformly distributed in network and they are stationary after deployment.

- Nodes are homogeneous and have the same capabilities. Each node is assigned a unique identifier (ID).

- All nodes can send data to Gateway nodes.

- All nodes have the information of gateway nodes locations via an initial broadcast message. 
- 10 Gateway nodes are dispersed in same sensor field at left most upper corner of the network area.

- Gateway nodes have the information of location of BS.

- Gateway Nodes have endless battery power means their batteries can be recharged.

- Gateway nodes have only two responsibilities, one is received data from cluster heads and second is transmit the data to BS.

- A gateway node can connect with only one $\mathrm{CH}$ node. The protocol limits that a gateway node can connect with only one $\mathrm{CH}$ node.

- Each Sensor node has the same initial power.

- In the first round, each node has a probability p of becoming the cluster head..

- Data compression is done by the Cluster head.

- Energy of transmission depends on the distance (source to destination) and data size.

\subsection{Proposed Algorithm}

The proposed algorithm works in rounds. Each round performs these following steps:

1. Periodically the base station starts a new round by incrementing the round number.

2. Selects cluster heads on the basis of leach protocol with probability 0.1 and the $\mathrm{CH}$ should not be more than 10 in number, in each round. In each round a sensor node elects itself as a cluster head by selecting a random number to compare to the threshold value. The threshold $T(n)$ is set as: $T(n)=\{\mathrm{P} / 1-\mathrm{P} *(\mathrm{r} \bmod 1 / \mathrm{P})\}$ if $\mathrm{n}$ belongs to $\mathrm{G}$, if not its 0 . $\mathrm{P}$ is the desired percentage of cluster heads, $r$ is the current round, and $G$ is the set nodes that have not been cluster heads in the last $(1 / \mathrm{P})$ rounds.

3. As soon as a $\mathrm{CH}$ is formed, it selects a gateway node which lies closest to it.

4. Make Clusters by allocating the cluster head to each node of the network on the basis of minimum distance between nodes to Cluster head $(\mathrm{CH})$.

5. Sensor nodes wake up, senses data, and forwards sensed data to respective CHs.

6. The CHs aggregates data receiving from all cluster members and then send data to the gateway nodes on the basis of one-to-one communication.

7. Now the gateway nodes further send the data to the BS and protocol goes in next round till the last round is not encountered.

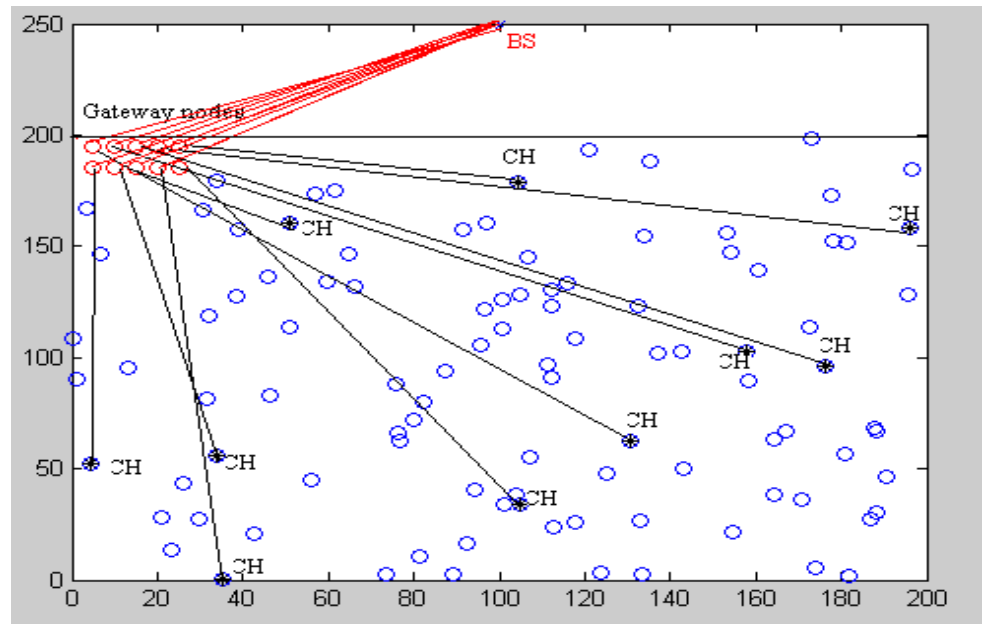

Figure 1. Model view of the proposed scheme 
International Journal of Wireless \& Mobile Networks (IJWMN) Vol. 4, No. 4, August 2012

\subsection{Flowchart of Proposed Algorithm}

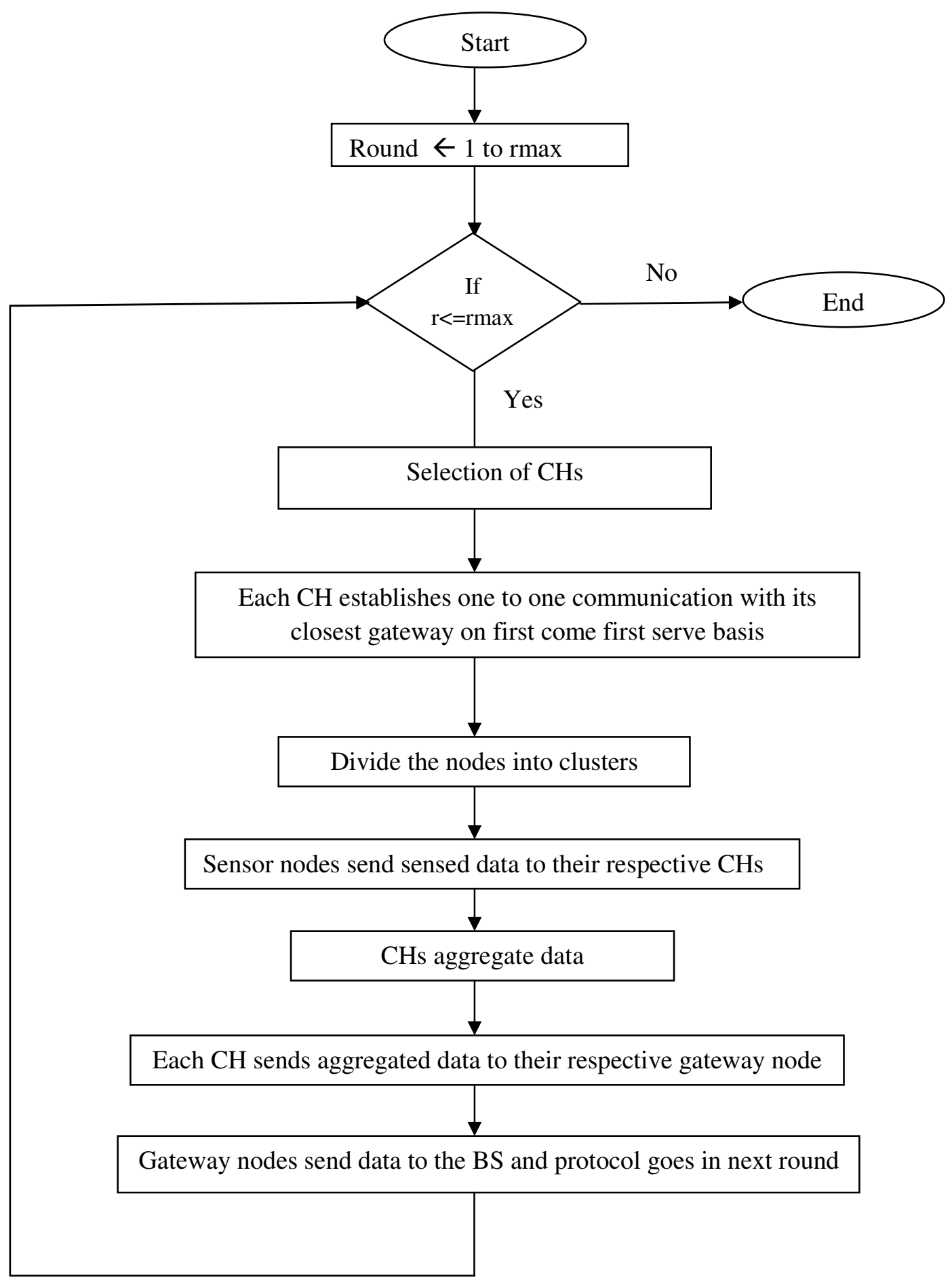

Figure 2. Flow Chart of Proposed Algorithm 
International Journal of Wireless \& Mobile Networks (IJWMN) Vol. 4, No. 4, August 2012

\subsection{Pseudo Code}

- $\quad$ for each round

- $\quad / /$ Choosing Cluster Head

- $\quad$ Threshold is set to $(\mathrm{P} /(1-\mathrm{P} *($ round \% $1 / \mathrm{P})))$

- $\quad$ for each node

- $\quad\{$ if number of cluster $<=10 \& \&$ energy of node $>0$

- $\quad$ Assign a random number

- $\quad\{$ if (random number $<$ threshold value) $\& \&$ (the node has not been cluster head)

- $\quad$ Node is Cluster head //assign node id to cluster head list

- Increment cluster head count //a new cluster head has been added

- $\quad$ Else go to the next node\}

- $\quad$ Else go to the next node\}

$\bullet \quad\}$

$\bullet$

- // CHs connecting to gateway nodes

- $\{$ for each cluster head

- $\quad$ for all gateway nodes

- cluster head coordinate $\mathrm{x}$ is assigned to $\mathrm{x} 1$

- cluster head coordinate $y$ is assigned to $y 1$

- $\quad$ if gateway node flag is false

- $\quad$ gateway node coordinate $\mathrm{x}$ is assigned to $\mathrm{x} 2$

- $\quad$ gateway node coordinate $y$ is assigned to $y 2$

- $\quad\{$ if it is the first gateway node

- $\quad$ the distance between cluster head and gateway node is the least distance

- $\quad$ gateway node id is assigned as closest gateway node to cluster head

- $\quad\}$

- $\quad$ else

- Distance between cluster head and current gateway node is current

distance

- $\quad\{$ if current distance $<$ least distance

- $\quad$ Current distances is now assigned to least distance

- $\quad$ Cluster gateway node id is assigned as closest gateway node to cluster

head

- Gateway node flag is set true

- $\}$

- $\quad\}$

- $\quad$ Else go to next gateway node

- $\}$

- $\}$

-

- // Generating Clusters

- $\quad$ For each node

- $\quad$ if node is a cluster head

- go to next node 
International Journal of Wireless \& Mobile Networks (IJWMN) Vol. 4, No. 4, August 2012

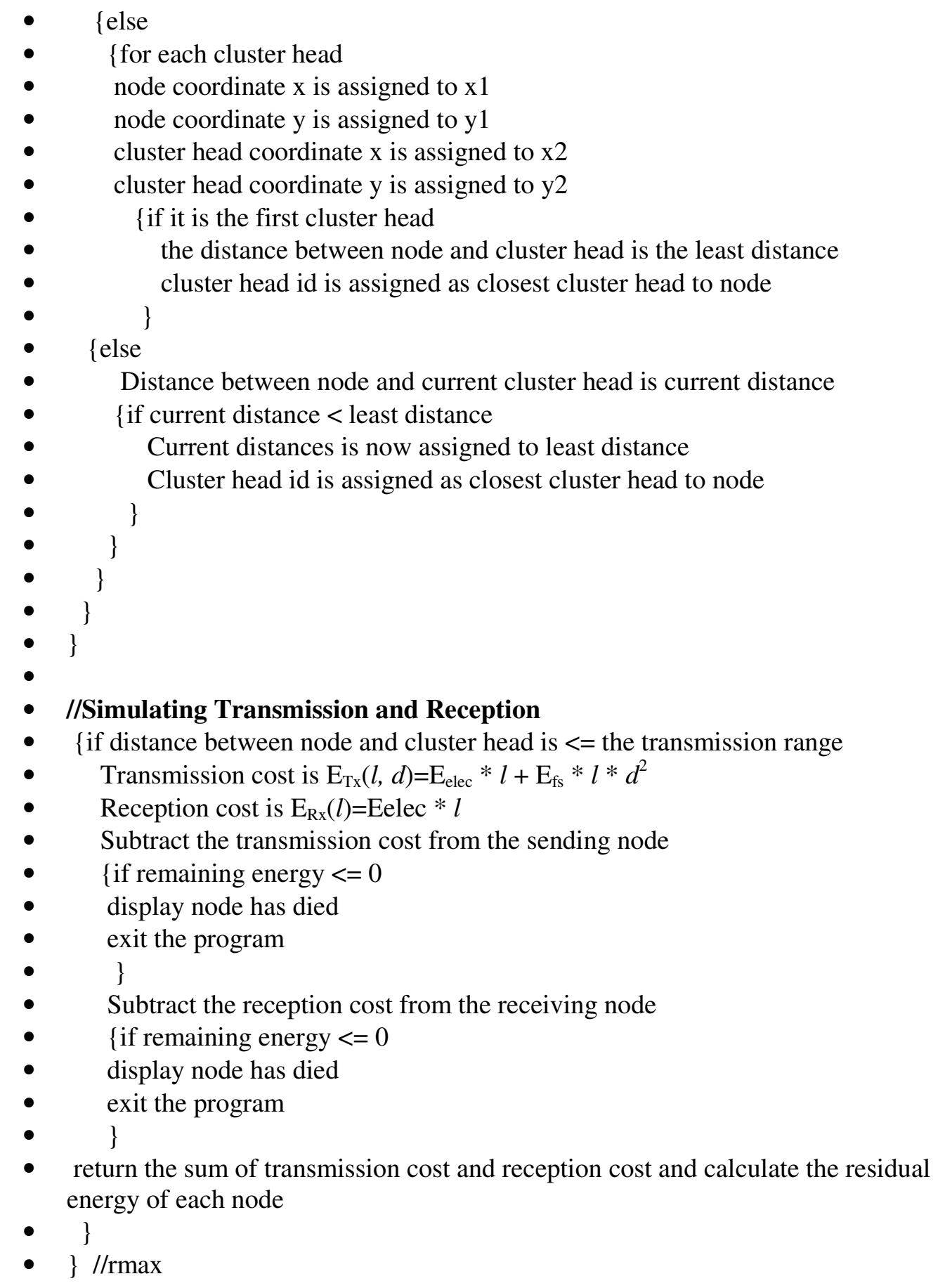

\section{Simulation and Performance Evaluations}

All simulations have been implemented using MATLAB. Assuming that 100 nodes are randomly distributed in field of 200x200 and the sink is located about 50m away from the field edge. The simulation parameters are given in Table 1 . The performance of the proposed multihop protocol scheme is compared with that of the single hop Leach protocol. 


\subsection{Energy Model for Communication}

We assume a simple model for the radio hardware energy dissipation where the transmitter dissipates energy to run the radio electronics and the power amplifier, and the receiver dissipates energy to run the radio electronics. For the experiments described here only the free space channel model is used. Thus, to transmit an $l$-bit message a distance $d$, the radio expends energy:

$$
\mathrm{E}_{\mathrm{Tx}}(l, d)=\left(l \mathrm{E}_{\mathrm{elec}}+l \mathrm{E}_{\mathrm{fs}} d^{2}\right)
$$

To receive this message, the radio expends energy:

$$
\mathrm{E}_{\mathrm{Rx}}(l)=l \mathrm{E}_{\text {elec }}
$$

\subsection{Simulation Parameter}

Table 1. Simulation Parameters

\begin{tabular}{|l|l|}
\hline Parameter & Values \\
\hline Simulation Round & 2000 \\
\hline Sink Location & $(100,250)$ \\
\hline Network Size & $200 \times 200$ \\
\hline Number of nodes & 100 \\
\hline Number of Gateway nodes & 10 \\
\hline CH probability & 0.1 \\
\hline Fusion rate $(c c)$ & 0.6 \\
\hline Initial node power & 0.5 Joule \\
\hline Nodes Distribution & Nodes are uniformly distributed \\
\hline Control Packet Size & 500 bits \\
\hline Data Packet size & 4000 bits \\
\hline Energy dissipation $($ Efs $)$ & $10^{*} 0.000000000001$ Joule \\
\hline Energy for Transmission $\left(\mathrm{E}_{\mathrm{TX}}\right)$ & $50^{*} 0.000000000001$ Joule \\
\hline Energy for Reception $\left(\mathrm{E}_{\mathrm{RX}}\right)$ & $50 * 0.000000000001$ Joule \\
\hline Energy for Data Aggregation $(\mathrm{EDA})$ & $5 * 0.000000000001$ Joule \\
\hline
\end{tabular}

\subsection{Simulation Results}

\subsubsection{Network Life Time}

When a node is dead in the network it'll not be the part of the network. It shows that if a dead node occurs in early rounds of the algorithm, this may affect lifespan of the network or drag towards the early dead of all nodes. Table 2 shows the simulation results of the two schemes. Fig. 3 concludes that in the proposed algorithm, the first node dies later in the network. 
International Journal of Wireless \& Mobile Networks (IJWMN) Vol. 4, No. 4, August 2012

Table 2. Network Life Time (First node dead)

\begin{tabular}{|c|c|c|}
\hline \multirow{2}{*}{$\begin{array}{c}\text { No. of } \\
\text { Simulation } \\
\text { Runs }\end{array}$} & \multicolumn{2}{|c|}{$\begin{array}{c}\text { Round Number when first } \\
\text { node dies }\end{array}$} \\
\cline { 2 - 3 } & Leach & $\begin{array}{c}\text { Proposed } \\
\text { scheme }\end{array}$ \\
\hline 1 & 302 & 365 \\
\hline 2 & 312 & 395 \\
\hline 3 & 298 & 343 \\
\hline 4 & 282 & 384 \\
\hline 5 & 309 & 343 \\
\hline 6 & 272 & 382 \\
\hline 7 & 262 & 329 \\
\hline 8 & 294 & 348 \\
\hline 9 & 261 & 380 \\
\hline 10 & 295 & 362 \\
\hline
\end{tabular}

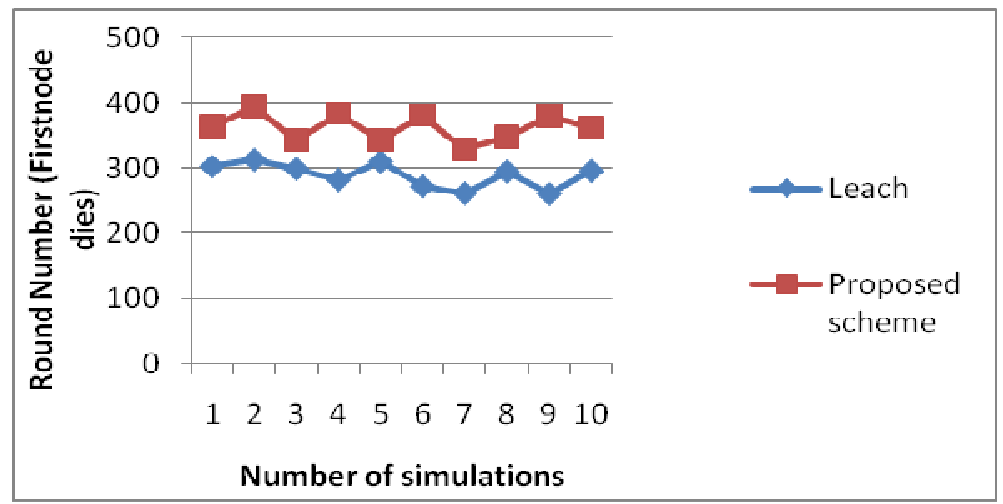

Figure 3. Network Life Time (First node dead) v/s No. of Simulation run

\subsubsection{Network Lifetime with Number of Alive Nodes}

More alive nodes contribute to the increase in network life time.Table 3 and Figure 4 show the number of nodes alive in the network with the increase in number of rounds. It is vivid that the lifetime of WSN using multi-hop proposed scheme is better compared to Leach Protocol.

Table 3. Network Life Time with Number of Alive nodes

\begin{tabular}{|c|c|c|}
\hline \multirow{2}{*}{$\begin{array}{c}\text { Round } \\
\text { Number }\end{array}$} & \multicolumn{2}{|c|}{ Number of Alive Nodes } \\
\cline { 2 - 3 } & Leach & $\begin{array}{c}\text { Proposed } \\
\text { scheme }\end{array}$ \\
\hline 100 & 100 & 100 \\
\hline 300 & 92 & 100 \\
\hline 600 & 59 & 76 \\
\hline 900 & 27 & 46 \\
\hline
\end{tabular}


International Journal of Wireless \& Mobile Networks (IJWMN) Vol. 4, No. 4, August 2012

\begin{tabular}{|c|c|c|}
\hline 1200 & 24 & 38 \\
\hline 1500 & 15 & 30 \\
\hline 1800 & 13 & 21 \\
\hline 2100 & 5 & 17 \\
\hline
\end{tabular}

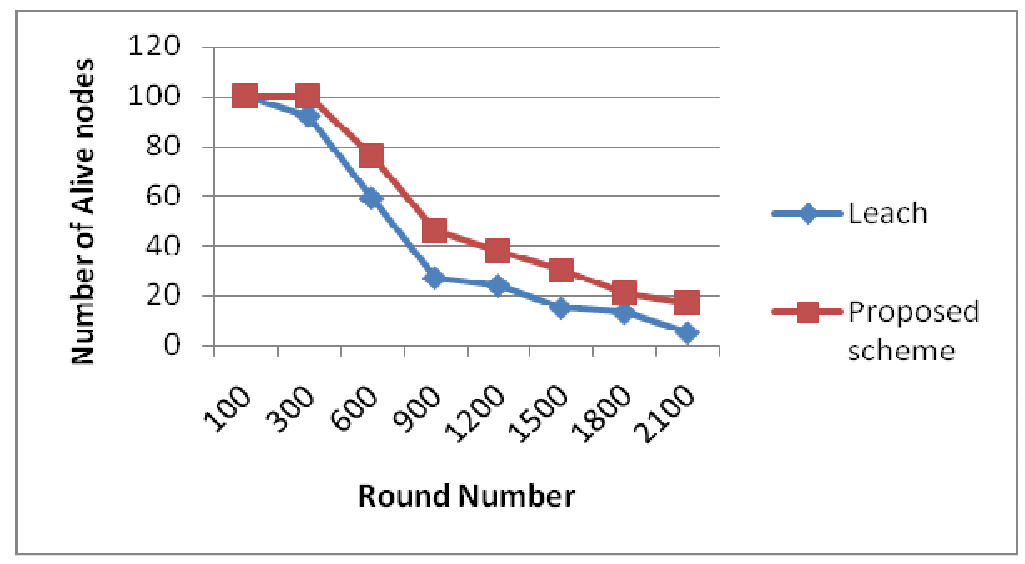

Figure 4. Network Life Time (Alive nodes) v/s Number of Rounds

\subsubsection{Network Life Time with Varying Packet Size}

Even on varying the packet size the network lifetime for the proposed algorithm remains better than that of the Leach. Table 4 and Fig.5 show the results on comparison.

Table 4. Network Life Time with different Packet Size

\begin{tabular}{|c|c|c|}
\hline \multirow{2}{*}{ Packet Size } & \multicolumn{2}{|c|}{$\begin{array}{c}\text { Round Number when first } \\
\text { node dies }\end{array}$} \\
\cline { 2 - 3 } & leach & $\begin{array}{c}\text { Proposed } \\
\text { Scheme }\end{array}$ \\
\hline 10000 & 111 & 136 \\
\hline 9000 & 133 & 148 \\
\hline 8000 & 134 & 169 \\
\hline 7000 & 156 & 220 \\
\hline 6000 & 193 & 274 \\
\hline 5000 & 216 & 342 \\
\hline 4000 & 295 & 380 \\
\hline 3000 & 463 & 633 \\
\hline 2000 & 615 & 744 \\
\hline 1000 & 1179 & 1265 \\
\hline
\end{tabular}


International Journal of Wireless \& Mobile Networks (IJWMN) Vol. 4, No. 4, August 2012

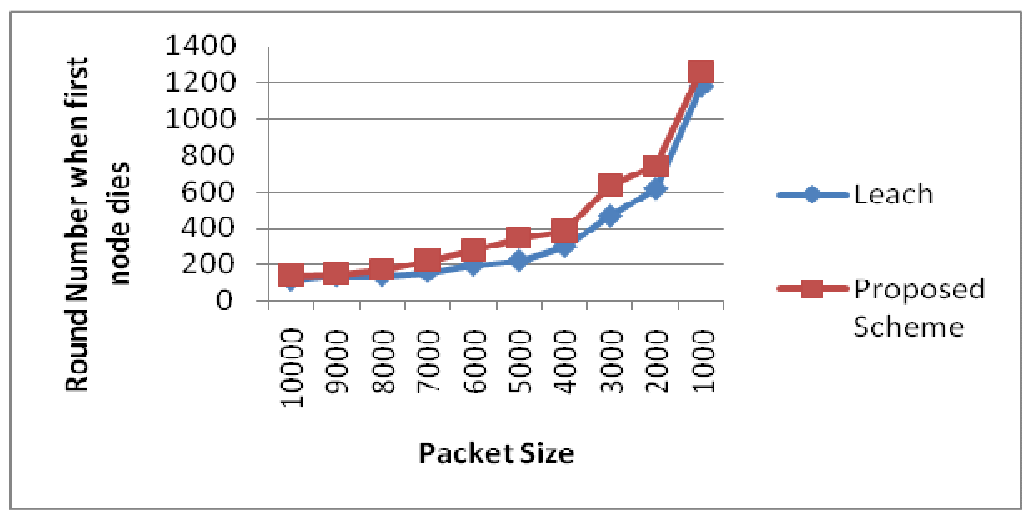

Figure 5. Network Life Time with varying packet size

\section{ConClusions}

Energy consumption is the main design issue in routing of wireless Sensor Network. We concluded that energy consumed for single hop transmission is more than multi-hop transmission for long distances. A new multi-hop routing protocol for the homogeneous wireless sensor networks has been presented and the performance of the system is evaluated to minimize the energy consumption and increase the life time of sensor network. The simulation results reveal that the LEACH protocol consumes more energy and the network has shorter lifetime than proposed multi-hop protocol with gateway nodes. We have determined the impact of packet length on the network lifetime. Finally, simulation results indicate that proposed protocol can more efficiently balance energy consumption of an entire network and thus extends the network lifetime. The proposed protocol is for the homogeneous network and we propose to extend this work for heterogeneous network in future work.

\section{REFERENCES}

[1] Wireless sensor network survey Jennifer Yick, Biswanath Mukherjee, Dipak Ghosal, Department of Computer Science, University of California, Davis, CA 95616, United States

[2] H. W. Kim, H. S. Seo(2010), "Modeling of Energy-efficient Applicable Routing Algorithm in WSN", International Journal of Digital Content Technology and its Applications, vol. 4, no. 5, pp.13-22.

[3] W. B. Heinzelman, A. P. Cnandrakasan,(2002) "An application-specific protocol architecture for wireless microsensor networks," IEEE Transactions on Wireless Communications, vol. 1, no. 4, pp.660-670.

[4] http://www.mathworks.in

[5] Wendi Rabiner Heinzelman (2000)."Energy-Efficient Communication Protocol for Wireless Microsensor Networks". In Proceeding of the $33^{\text {rd }}$ Hawaii International Conference on System Sciences,pp1-10

[6] Ankita Joshi, Lakshmi Priya.M "A Survey of Hierarchical Routing Protocols in Wireless Sensor Network", International Conference on Information Systems 
International Journal of Wireless \& Mobile Networks (IJWMN) Vol. 4, No. 4, August 2012

[7] Rajashree.V.Biradar , Dr. S. R. Sawant , Dr. R. R. Mudholkar, Dr. V.C .Patil (2011)" Inter-Intra Cluster Multihop-LEACH Routing In Self-Organizing Wireless Sensor Networks", International Journal of Research and Reviews in Computer Science (IJRRCS) Vol. 2, No. 1.

[8] Muhamnmad Omer Farooq, Abdul Basit Dogar, Ghalib Asadullah Shah (2010)" MR-LEACH: Multi-hop Routing with Low Energy Adaptive Clustering Hierarchy", Fourth International Conference on Sensor Technologies and Applications.

[9] Guihai Chen - Chengfa $\mathrm{Li} \cdot \mathrm{Mao} \mathrm{Ye} \cdot \mathrm{JieWu}$, An unequal cluster-based routing protocol in wireless sensor networks", Wireless Netw DOI 10.1007/s11276-007-0035-8

[10] Seema Bandyopadhyay and Edward J. Coyle," An Energy Efficient Hierarchical Clustering Algorithm for Wireless Sensor Networks".

[11] F. Akyildiz, W. Su, (2002) "A survey on sensor networks", Communications Magazine, IEEE, vol. 40, no. 8, pp.102-114.

\section{Authors}

S.Taruna is an active researcher in the field of communication and mobile network, currently working as Assistant Professor in Department of Computer Science at Banasthali University (Rajasthan), India. She has done M.Sc from Rajasthan University and her $\mathrm{PhD}$ is in progress from Banasthali University (Rajasthan), India. She has presented many papers in National and International Conferences and published 4 papers in various journals.

Rekha Kumawat received B.E degree in Computer Science and Engineering from University of Rajasthan, Jaipur in 2008. She is pursuing her M.Tech degree in Computer Science from Banasthali University, Rajasthan. Her research interests include computer networks, wireless ad hoc and sensor networks.

Prof. G. N. Purohit is a Professor in Department of Mathematics \& Statistics at Banasthali University (Rajasthan). Before joining Banasthali University, he was Professor and Head of the Department of Mathematics, University of Rajasthan, Jaipur. He had been Chief-editor of a research journal and regular reviewer of many journals. His present interest is in O.R., Discrete Mathematics and Communication networks. He has published around 40 research papers in various journals.
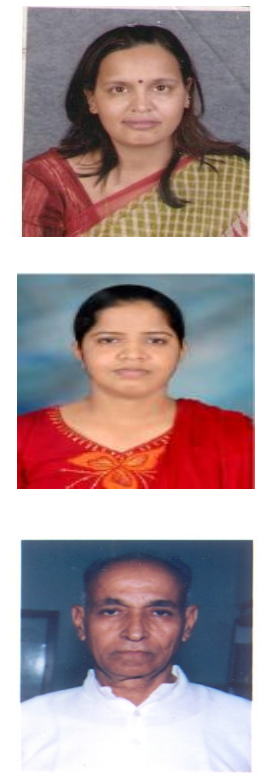